\title{
Aspectos dos transtornos mentais comuns ao puerpério
}

\author{
Aspects of mental disorders common to the puerperium
}

Aspectos de los trastornos mentales comunes al puerperio

Mariana Rodrigues Assef ${ }^{1 *}$, Ana Clara Machado Barina1, Ana Paula Pereira Martins², João Guilherme de Oliveira Machado ${ }^{3}$, Luana Oliveira Amado ${ }^{4}$, Lubiane de Toledo ${ }^{5}$, Ludmila Limpias Terrazas Binkowski ${ }^{6}$, Maria Carolina Álvares Correia ${ }^{7}$, Tiago Picolo Fernandes ${ }^{8}$, Gustavo Fonseca Genelhu Soares ${ }^{9}$.

\section{RESUMO}

Objetivo: Descrever e discutir as principais síndromes psiquiátricas que podem ocorrer no período puerperal, identificando seus fatores causadores mais comuns. Revisão Bibliográfica: O puerpério, período pós gestacional, compreende um momento de mudanças bruscas que incluem alterações hormonais, biológicas e sociais, configurando uma maior vulnerabilidade para o aparecimento de transtornos psíquicos. Dentre esses, destacam-se a disforia pós-parto, a depressão puerperal e a psicose puerperal. Estudos mostraram que a disforia pós-parto foi observada em 50-85\% das mulheres, sendo considerada um fenômeno fisiológico que pode interromper os cuidados com o bebê, aumentando o risco de desenvolver a depressão pós-parto e prejudicando ainda o desenvolvimento infantil. A depressão pós-parto possui as mesmas características clínicas do transtorno depressivo maior, podendo cursar com isolamento social, fadiga, instabilidade de humor, inconstância emocional, choro e sentimento de culpa e incapacidade. Já a psicose puerperal é a forma mais grave, considerada uma emergência psiquiátrica. Considerações finais: Tais transtornos quando subnotificados ou não tratados podem ter repercussões irreversíveis. Nesse contexto, é necessário reforçar a importância do acolhimento e atendimento humanizado às puérperas e seus filhos bem como o diagnóstico precoce desses quadros.

Palavras-chave: Puerpério, Pós-parto, Ansiedade, Saúde mental.

\begin{abstract}
Objective: To describe and discuss the main psychiatric syndromes that can occur in the puerperal period, identifying their most common causative factors. Bibliographic Review: The postpartum period, postpregnancy period, includes a moment of sudden changes that include hormonal, biological and social changes, configuring a greater vulnerability for the appearance of psychic disorders. Among these, postpartum dysphoria, puerperal depression and puerperal psychosis stand out. Studies have shown that postpartum dysphoria was observed in $50-85 \%$ of women, being considered a physiological phenomenon that can interrupt baby care, increasing the risk of developing postpartum depression and also impairing child development. Postpartum depression has the same clinical characteristics a major depressive disorder, which can lead to
\end{abstract}

\footnotetext{
1 Universidade Vila Velha (UVV), Vila Velha - ES. *E-mail: mariana.assef@hotmail.com

2 Universidade do Estado de Minas Gerais (UEMG), Divinópolis - MG.

${ }^{3}$ Faculdade Brasileira (MULTIVIX), Vitória - ES.

${ }^{4}$ Faculdade Ciências Médicas de Minas Gerais (FCMMG), Belo Horizonte - MG.

${ }^{5}$ Centro Universitário de Maringá (Unicesumar), Maringá - PR.

${ }^{6}$ Universidade Luterana do Brasil (ULBRA), Canoas - RS.

${ }^{7}$ Instituto Metropolitano de Ensino Superior (IMES/UNIVAÇO), Ipatinga - MG.

8 Universidade Nove de Julho (UNINOVE), Bauru - SP.

${ }^{9}$ Centro Universitário de Caratinga (UNEC), Caratinga - MG.
} 
social isolation, fatigue, mood instability, emotional inconstancy, crying and feelings of guilt and incapacity. Puerperal psychosis is the most serious form, considered a psychiatric emergency. Final considerations: Such disorders when underreported or untreated can have irreversible repercussions. In this context, it is necessary to reinforce the importance of welcoming and humanized care for mothers and their children, as well as the early diagnosis of these conditions.

Key words: Postpartum period, Postpartum, Anxiety, Mental health.

\section{RESUMEN}

Objetivo: Describir y discutir los principales síndromes psiquiátricos que pueden ocurrir en el puerperio, identificando sus factores causales más comunes. Revisión bibliográfica: El posparto, pos gestacional, incluye un momento de cambios bruscos que incluyen cambios hormonales, biológicos y sociales, configurando una mayor vulnerabilidad para la aparición de trastornos psíquicos. Entre estos destacan la disforia posparto, la depresión puerperal y la psicosis puerperal. Los estudios han demostrado que la disforia posparto se observó en el $50-85 \%$ de las mujeres, considerándose un fenómeno fisiológico que puede interrumpir el cuidado del bebé, aumentando el riesgo de desarrollar depresión posparto y también perjudicando el desarrollo infantil. La depresión posparto tiene las mismas características clínicas que el trastorno depresivo mayor, lo que puede provocar aislamiento social, fatiga, inestabilidad del humor, inconstancia emocional, llanto y sentimientos de culpa e incapacidad. La psicosis puerperal es la forma más grave, considerada una emergencia psiquiátrica. Consideraciones finales: Estos trastornos, cuando no se notifican o no se tratan, pueden tener repercusiones irreversibles. En este contexto, es necesario reforzar la importancia de la atención acogedora y humanizada para las madres y sus hijos, así como el diagnóstico precoz de estas condiciones.

Palabras clave: Periodo posparto, Posparto, Ansiedad, Salud mental.

\section{INTRODUÇÃO}

Os agravos à saúde mental são doenças de interesse geral a serem estudadas, estando os transtornos depressivos unipolares com a quarta posição dos principais determinantes da carga global de doenças. $O$ impacto negativo da síndrome depressiva na saúde humana é mais frequentemente observado entre as mulheres, que apresentam um risco duas vezes maior que os homens de desenvolver depressão, sendo esta diferença ainda mais perceptível na fase da vida em que elas se veem responsáveis pelo cuidado de seus filhos (WORLD HEALTH ORGANIZATION (WHO), 2018).

No puerpério, definido como o período que ocorre após o nascimento do concepto até o tempo de seis a oito semanas após o parto, a mulher passa por mudanças bruscas dos níveis hormonais, alterações biológicas, sociais e psicológicas. Nessa fase, muitas vezes, a mãe precisa reorganizar e adaptar sua vida, sofrendo frequentemente de privação do sono e baixa autoestima, predispondo a uma maior vulnerabilidade para o aparecimento de diversos transtornos psíquicos (SLOMIAN J, et al., 2019).

Ao examinar uma puérpera deve-se, inicialmente, verificar não somente sua situação clínica, como também realizar uma breve avaliação do seu estado psíquico e emocional. A importância dessa avaliação se justifica uma vez que os transtornos psiquiátricos puerperais, suscetíveis nessa fase, trazem repercussões negativas tanto para a mãe e familiares quanto para o filho, podendo interferir, inclusive, no desenvolvimento da criança, causando impactos negativos desde a infância até a sua vida adulta (GOMES GF e SANTOS AV, 2017).

Dentre os transtornos psíquicos puerperais conhecidos, os mais comuns são a depressão pós-parto, disforia pós-natal e a psicose puerperal (TEIXEIRA CS, et al., 2019). A Depressão Pós-Parto (DPP) possui as mesmas características clínicas do episódio depressivo maior, que segundo a American Psychiatric Association (APA) (2014), incluem no mínimo cinco sintomas presentes por pelo menos duas semanas, com humor deprimido e perda de interesses nas atividades necessariamente, além de labilidade emocional, 
insônia, ideias suicidas, ansiedade e sentimento de culpa. Esse transtorno repercute negativamente na interação entre mãe e bebê, promovendo desgaste progressivo nas relações familiares entre a puérpera e seus familiares, trazendo diversas alterações emocionais, cognitivas, comportamentais e físicas na saúde materna e da criança (CARLESSO GP, et al., 2014)

A disforia puerperal é também conhecida como blues puerperal e se apresenta tipicamente com sintomas leves de instabilidade emocional, alterações de humor, choro e ansiedade, mas não interferem na capacidade da mãe de cuidar de si e do recém-nascido (SADOCK BJ, et al., 2017). Já a psicose pós-parto, segundo o Manual Diagnóstico e Estatístico de Transtornos Mentais (DSM-5), é a forma mais grave dos transtornos psíquicos do puerpério, sendo considerada uma emergência psiquiátrica. A mulher apresenta sintomas de agitação, insônia, irritabilidade e humor depressivo, além de sintomas delirantes e alucinações auditivas relacionadas à criança, aumentando os riscos para o infanticídio e o suicídio se não tratadas (FABIANO $\mathrm{F} \mathrm{e}$ HASLAM N, 2020).

Dessa forma pode se notar que o cuidado à mulher e ao recém-nascido no pós-parto imediato e nas primeiras semanas após o parto é fundamental para a saúde materna e do bebê e o acolhimento deve ser realizado por toda equipe de saúde. Além disso, é de suma importância que a equipe de saúde procure acolher a puérpera da melhor forma possível, propondo para ela um plano terapêutico, visto que a gestação muda o corpo da mulher, sua rotina, seus hábitos e o cuidado integral e humanizado é indispensável nessa etapa da vida (GOMES GF e DOS SANTOS AP, 2017).

Nesse contexto, esse estudo visa descrever e discutir as principais síndromes psiquiátricas que podem ocorrer no período puerpério, identificando os fatores causadores dessa patologia e reforçar a importância do acolhimento e atendimento humanizado às puérperas e seus bebês nesse período.

\section{REVISÃO BIBLIOGRÁFICA}

A gravidez é um evento desejado e planejado para algumas mulheres, marcado por alegrias e realizações, entretanto, em alguns casos, podem surgir sentimentos conflituosos tanto em relação ao recém-nascido quanto à própria vida da gestante (ALMEIDA NM e ARRAIS AR, 2016). O período gravídico-puerperal é considerado um evento estressante para muitas mulheres e diversos fatores corroboram para o surgimento dos transtornos psíquicos sejam eles biológicos, psicológicos ou até mesmo a associação destes com o meio cultural e social (MOURA E, et al., 2011).

Entre esses fatores, a falta de apoio por parte do parceiro ou mesmo da família, gestação não planejada, transtornos afetivos, dificuldade de engravidar ou na amamentação, vivência de perdas, nascimento de bebês com anomalias, prematuridade, ser solteira ou divorciada e gestar um bebê do sexo oposto ao planejado são exemplos de fatores psicossociais possivelmente causadores desses transtornos. As alterações nos níveis hormonais, bem como a resposta ao estresse também se relacionam com a patogênese. Vale salientar que antecedentes familiares e pessoais de transtornos psiquiátricos são fatores de risco para o desenvolvimento de tais patologias (BECKER M, et al., 2016).

As doenças psiquiátricas pós-parto são pouco reconhecidas e estudadas. Os transtornos psíquicos puerperais são definidos como doenças mentais com início no primeiro ano após o parto, e que se manifestam por desequilíbrios do humor psicóticos e não psicóticos. Tais transtornos, quando subdiagnosticados e não tratados, fazem com que as puérperas se sintam incapazes de exercer o papel materno, sofrendo com sentimento de culpa e variações de humor (FROTA C, et al., 2020).

Entre os principais transtornos destacam-se: a disforia pós-parto, caracterizado como um distúrbio psíquico leve e transitório; a Depressão puerperal, que pode ser um transtorno moderado a severo com início insidioso; e, por fim, a Psicose puerperal, que é um distúrbio de humor psicótico apresentando perturbações mentais graves (GONÇALVES M, 2017).

Mesmo a gestação sendo tipicamente considerada um período de bem-estar emocional, a incidência de transtornos mentais comuns durante a gestação, no Brasil, é de 33,6\%. Estima-se que a depressão puerperal ocorra em $10-20 \%$ das puérperas, sendo que, na adolescência, foi encontrada uma prevalência até duas 
vezes mais elevada. A disforia pós-parto foi observada em $50-85 \%$ das mulheres pesquisadas, e a psicose pós-parto em apenas 0,1-0,2\% (LOPES R, et al., 2019).

A disforia pós-parto, ou tristeza da maternidade ou "baby blues" é considerada um fenômeno fisiológico, com um pico característico entre três e cinco dias no pós-parto, mas os modelos e critérios para diagnóstico da tristeza materna não foram bem estabelecidos (ZANARDO V, et al., 2019). A apresentação clínica se baseia em mudanças repentinas no temperamento, choro inexplicável, irritabilidade e impaciência. Além disso, a paciente apresenta falta de sono, crises de choro, ansiedade, solidão e sensação de vulnerabilidade (SADOCK BJ, et al., 2017).

Esse transtorno de humor pode interromper os cuidados necessários com o bebê, aumentando o risco de sintomas de depressão pós-parto e prejudicando as interações mãe-filho, afetando, assim, o desenvolvimento infantil. A causa mais provável atribuída a esse transtorno é a mudança hormonal repentina após o parto. Dessa maneira, as mulheres que são mais sensíveis a essas mudanças têm maior incidência de tristeza materna do que as mulheres que não são (REZAIE-KEIKHAIE K, et al., 2020).

Os babys blues são um conjunto de sintomas psicossomáticos temporários, não sendo considerado uma condição patológica. Devido a sua resolução espontânea, não há a necessidade de terapia farmacológica. No entanto, pode ser um fator de risco para o desenvolvimento de depressão pós-parto. Por isso, um acompanhamento psiquiátrico com essas mulheres é recomendado (GERLI S, et al., 2019).

A Depressão Pós-Parto (DPP), por sua vez, é uma condição que se inicia dentro dos primeiros 12 meses após o parto, principalmente entre a quarta e a oitava semana, e afeta cerca de 10 a $15 \%$ das mulheres. Esse quadro pode trazer consequências negativas na relação entre a mãe e o recém-nascido e no desenvolvimento emocional, cognitivo e intelectual do filho, além de proporcionar menor qualidade de vida e de bem-estar da mulher. Assim, se faz necessário o diagnóstico precoce e a detecção dos possíveis fatores associados (FERREIRA C, et al., 2018).

A prevalência da depressão após o período gestacional varia de acordo com os países (sendo maior naqueles menos desenvolvidos), com o perfil socioeconômico, com o nível de escolaridade, com a convivência entre o casal e com os hábitos maternos relacionados a álcool e tabaco (HARTMANN JM, et al., 2017). Estudos identificaram que o relacionamento entre a mulher e seu parceiro possui grande influência na incidência da depressão pós-parto. Deficiência na intimidade e no suporte no casamento, histórico prévio de depressão, ansiedade durante a gravidez e falta de apoio social são considerados fatores de risco (JEONG Y-J, et al., 2021).

As investigações acerca dessa condição, o reconhecimento e interpretação do comportamento materno e a identificação precoce dos sintomas que podem indicar a depressão são de suma importância pois contribuem para o planejamento de ações e condutas que melhorem a saúde da puérpera. Os sintomas apresentados durante a depressão pós-parto consistem no isolamento social, fadiga, instabilidade de humor, inconstância emocional, choro e tristeza, sentimento de culpa e inutilidade, sensação de incapacidade de cuidar do recém-nascido e desligamento emocional e afetivo para com o bebê e outros membros da família (BOSKA GA, et al., 2016).

Outro fator relevante no âmbito psicológico da mulher é a auto aceitação do seu corpo e das mudanças físicas ocasionadas pela gravidez. Esse período é marcado por mudanças específicas no corpo, como ganho de peso e alterações na forma e na pele e, devido à imagem estereotipada feminina do corpo ideal que é disseminada pela mídia, muitas mulheres apresentam insatisfação corporal e baixa autoestima. Atitudes negativas em relação à maternidade associadas à não adaptação das mulheres grávidas às mudanças biológicas, psicológicas e sociais são determinantes para o surgimento de sintomas depressivos (BASISTA HP, et al., 2020).

Segundo pesquisas, foi feita uma associação entre a depressão pós-parto e a amamentação. O processo de amamentação é fundamental para fornecer ao bebê os nutrientes necessários para seu desenvolvimento saudável e para promover a conexão entre mãe e filho. Sua duração é recomendada durante os seis primeiros meses de vida da criança, entretanto, ela pode ser afetada pela saúde mental da mãe e pela falta de apoio 
da família e profissionais da equipe de saúde. Estudos concluíram que o aumento dos níveis de depressão da mulher causa diminuição na sua capacidade de amamentar. Por outro lado, a amamentação amplia os processos hormonais que protegem as mães da depressão pós-parto, uma vez que atenuam a liberação de cortisol em resposta a situações de estresse (MERCAN Y e SELCUK KT, 2021).

O diagnóstico da depressão pós-parto pode ser baseado na Escala de Depressão Pós-Parto de Edimburgo (EPDS), um instrumento de autopreenchimento, composto por 10 itens pontuados entre 0 e 3 . Esse método consiste no rastreamento dos sintomas depressivos, além de possibilitar uma reflexão emocional às mulheres (MORAES GA, et al., 2017). Outra maneira de reconhecer o estado depressivo materno é o Inventário de Depressão de Beck (BDI), questionário realizado, segundo os critérios da American Psychiatric Association (APA), no terceiro trimestre gestacional para auto avaliação de comportamentos. Ele é formado por 21 categorias de sintomas pontuadas de 0 a 3, assim como a Escala de Depressão Pós-Parto de Edimburgo (SCHIAVO RA e PEROSA GB, 2020).

O tratamento para a depressão pós-parto requer uma intervenção multidisciplinar. Uma vez que o diagnóstico foi concluído, estratégias psicossociais que incentivem o autocuidado, o apoio social e familiar e a redução dos impactos de situações de estresse são indicadas para qualquer paciente. Estudos até apresentaram a prática de exercícios aeróbicos como forma de promover uma pequena melhora no quadro. Em casos mais graves, entretanto, é necessário tratamento adicional, podendo ser medicamentoso, com o uso de antidepressivos e inibidores seletivos de recaptação de serotonina, ou não, como a Terapia CognitivoComportamental (TCC) e terapia interpessoal (STEWART DE e VIGOD SN, 2019).

Ainda dentre os transtornos psíquicos puerperais, a psicose puerperal é a manifestação mais grave de sintomas depressivos durante a gestação ou até duas semanas após o parto, sendo considerada uma emergência psiquiátrica de início repentino. O início dos sintomas é abrupto, com presença de alucinações, angústia, insônia, delírios, estado confusional e observação grave ou pensamentos delirantes relacionados ao bebê. Além disso, trata-se de um transtorno mental com capacidade de rápido agravamento, com variedade da intensidade dos sintomas, associado a uma incidência elevada de suicídio e infanticídio (VANDERKRUIK R, et al., 2017).

Estudos recentes associaram risco de psicose pós-parto à ocorrência prévia de transtornos mentais. Em mulheres primíparas, um fator de risco a ser considerado é a presença de transtorno bipolar, que indica risco alto de psicose puerperal (26\%) quando comparado a mulheres sadias $(0,1-0,2 \%)$. Na bipolaridade, o risco elevado de recidiva grave é selecionado ao transtorno bipolar tipo 1, com sintomas psicóticos ao invés do tipo 2, com episódios não psicóticos ou depressivos hipomaníacos. Mulheres com bipolaridade anterior ou psicose puerperal apresentam igualdade na recidiva pós-parto, entretanto, mulheres com psicose puerperal sofrem doenças mais graves. Por fim, a psicose puerperal pode, ainda, evoluir para depressão pós-parto (OLSEN MT, et. al., 2014; CANTWELL R, 2021).

O diagnóstico inclui fatores de risco e sintomatologia típica da psicose puerperal, ambas citadas. Apesar de não haver guidelines para a abordagem da patologia, quando está relacionada ao transtorno bipolar na gestação, o tratamento é contínuo quando não há estabilidade anterior sem a terapêutica farmacológica. A Eletroconvulsoterapia (ECT), é utilizada na refração terapêutica dos medicamentos, quando existe risco de suicídio e infanticídio, além de reduzir o período de sintomas e restabelecer a relação mãe-lactante. Em casos especiais, a internação pode ser necessária (CANTWELL R, 2021).

A investigação de transtornos mentais puerperais na atenção básica ainda é um desafio para os profissionais de saúde, com dificuldades inclusive no reconhecimento dos sintomas para elaboração de um diagnóstico e do tratamento. Considerando a alta prevalência dos distúrbios mentais durante o período pós gestacional e os riscos a eles associados, tanto para a mãe quanto para o bebê, é de suma importância que os profissionais de saúde com um olhar holístico e empático ajudem essas mulheres tanto na prevenção da gravidez indesejada, com o uso de métodos anticoncepcionais, quanto facilitando a passagem pela fase gravídica puerperal (COSTA DO, et al., 2018).

Defende-se, assim, a construção de uma assistência obstétrica integral, com profissionais sensíveis às questões psicossociais. A avaliação e o acompanhamento das gestantes são fundamentais, pois esse 
cuidado gestacional pode ser o único contato que esta mulher está tendo com os serviços médicos, alertando para possíveis intervenções na promoção da saúde da mesma. A humanização dos profissionais pode auxiliar no bom desenvolvimento emocional e no melhor prognóstico para essas gestantes (TEIXEIRA CS, et al 2019, COSTA DO, et al., 2018).

\section{CONSIDERAÇÕES FINAIS}

A partir da análise da literatura, verificou-se que, além das oscilações bioquímicas comuns na mulher após o parto, outros fatores como a privação de sono, baixa autoestima, ansiedade, falta de apoio do companheiro e da família, gestação não planejada, bebês com anomalias e antecedentes familiares contribuem para o desenvolvimento ou agravamento de doenças psiquiátricas. Entre essas doenças, observou-se que, em nível mais leve, tem-se a disforia pós-parto e em nível moderado a depressão pós-parto. A psicose puerperal, por sua vez, é a manifestação de maior gravidade de transtorno psiquiátrico puerperal. Dessa forma, ressalta-se a importância da atenção à mulher e ao recém-nascido neste período, com o acolhimento da equipe de saúde e oferecimento de tratamento integral e humanizado.

\section{REFERÊNCIAS}

1. ALMEIDA NM, ARRAIS AR. Programa de Prevenção à Depressão Pós-Parto Psicologia: Ciência e Profissão, 2016; 36 (4): 847-863.

2. AMERICAN PSYCHIATRIC ASSOCIATION. Manual diagnóstico e estatístico de transtornos mentais: DSM-5. 5.ed. Porto Alegre: Artmed, 2014; 992p.

3. BASISTA HP, et al. Body acceptance by pregnant women and their attitudes toward pregnancy and maternity as predictors of prenatal depression. International Journal of Environmental Research and Public Health, 2020; 17(24):9436

4. BECKER M, et al. Depression During Pregnancy and Postpartum. Current Psychiatry Reports. 2016; 18(3):32.

5. BOSKA GA, et al. Sintomas depressivos no período puerperal: identificação pela escala de depressão pós-parto de Edinburgh.Journal of Nursing and Health. 2016;1(1):38-50.

6. CANTWELL R. Transtorno mental na gravidez e no início do pós-parto. Anestesia, 2021; 76(4):76-83.

7. CARLESSO GP, et al. Análise da relação entre depressão materna e indicadores clínicos de risco para o desenvolvimento infantil. Revista CEFAC, 2015; 16(2): 500-510.

8. COSTA DO, et al. Transtornos mentais na gravidez e condições do recém-nascido: estudo longitudinal com gestantes assistidas na atenção básica. Ciência \& Saúde Coletiva, 2018; 23(3):691-700.

9. FABIANO F, HASLAM N. Diagnostic inflation in the DSM: A meta-analysis of changes in the stringency of psychiatric diagnosis from DSM-III to DSM-5. Clinical Psychology Review, 2020; 80:101889

10. FERREIRA C, et al. Postpartum depression: early detection and associated factors. Acta Obstétrica e Ginecológica Portuguesa, 2018; 12(4):262-267.

11. FROTA C, et al. A transição emocional materna no período puerperal associada aos transtornos psicológicos como a depressão pós-parto. Revista Eletrônica Acervo Saúde, 2020; 48 : e3237.

12. GERLI S, et al. Obstetric and psychosocial risk factors associated with maternity blues. Journal of Maternal-Fetal \& Neonatal Medicine, 2019; 34(8):1227-1232.

13. GOMES GF, SANTOS AP. Assistência de enfermagem no puerpério. Revista Enfermagem Contemporânea, 2017; 6(2):211-220.

14. GONÇALVES M. Transtornos mentais em pacientes em período puerperal. Part of the international journal of psychiatry, 2017; 15(7).

15. HARTMANN JM, et al. Depressão entre puérperas: prevalência e fatores associados. Cadernos de Saúde Pública 2017; 33(9):e00094016.

16. JEONG YJ, et al. Factors Influencing Quality of Life in Early Postpartum Women. International Journal of Environmental Research and Public Health, 2021; 18(6):2988.

17. LOPES R, et al. O período gestacional e transtornos mentais: evidências epidemiológicas. Humanidades \& tecnologia em revista, 2019; 19(1):35-54.

18. MERCAN Y, SELCUK KT. Association between postpartum depression level, social support level and breastfeeding attitude and breastfeeding self-efficacy in early postpartum women. PLOS ONE, 2021; 16(4): e0249538.

19. MORAES GA, et al. Screening and diagnosing postpartum depression: when and how? Trends Psychiatry Psychother, 2017; 39(1):54-61.

20. MOURA E, et al. Percepção materna sobre transtornos psiquiátricos no puerpério: implicações na relação mãe-filho. Revista Brasileira de Enfermagem, 2011; 64(3):445-50.

21. OLSEN M, et al. Birth order and postpartum psychiatric disorders. Bipolar disorders, 2014; 16(3): 300-307.

22. REZAIE-KEIKHAIE K, et al. Systematic Review and Meta-Analysis of the Prevalence of the Maternity Blues in the Postpartum Period. Journal of Obstetric, Gynecologic, \& Neonatal Nursing, 2020; 49(2):127-136. 
23. SADOCK BJ, et al. Compêndio de psiquiatria: ciência do comportamento e psiquiatria clínica. 11a ed. Porto Alegre: Artmed, 2017; 855p

24. SLOMIAN J, et al. Consequences of maternal postpartum depression: A systematic review of maternal and infant outcomes. Womens Health, 2019; 15:1-55.

25. STEWART DE, VIGOD SN. Postpartum Depression: Pathophysiology, Treatment, and Emerging Therapeutics. Annual Review of Medicine, 2019; 70:183-96.

26. SCHIAVO RA, PEROSA GB. Child development, maternal depression and associated factors: a longitudinal study. Paidéia (Ribeirão Preto), 2020; 30:e3012.

27. TEIXEIRA CS, et al. Aspectos da gestação e puerpério de mulheres com transtornos mentais. Revista de Enfermagem da UFPE, 2019; 13: e239705.

28. VANDERKRUIK $R$, et al. The global prevalence of postpartum psychosis: a systematic review. BMC Psychiatry. 2017;17(1):272.

29. WORLD HEALTH ORGANIZATION (WHO). WHO recommendations: intrapartum care for a positive childbirth experience. Geneva: World Health Organization, 2018. Disponível em: https://www.who.int/reproductivehealth/publications/intrapartum-care-guidelines/en/. Acessado em: 02 de maio de 2021.

30. ZANARDO V, et al. Maternity blues: a risk factor for anhedonia, anxiety, and depression components of Edinburgh Postnatal Depression Scale. The Journal of Maternal-Fetal \& Neonatal Medicine, 2019; $1-7$. 